\title{
Mechanoresponsive Elastomers Made with Excimer-Forming Telechelics
}

\author{
Derek J. Kiebala ${ }^{a}$ (i) \\ Zhiyuan Fan ${ }^{\mathrm{a}, \mathrm{b}}$ (D) \\ Celine Calvino ${ }^{\mathrm{a}, \mathrm{c}}$ (D) \\ Lauren Fehlmann ${ }^{a}$ (i) \\ Stephen SchrettI ${ }^{* a}$ (iD \\ Christoph Weder*a (D)

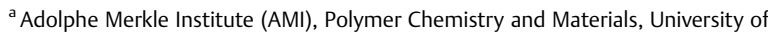 \\ Fribourg, Chemin des Verdiers 4, 1700 Fribourg, Switzerland \\ ${ }^{\mathrm{b}}$ Key Laboratory of Organic Optoelectronics and Molecular Engineering, Department \\ of Chemistry, Tsinghua University, Mengminweikeji Building South Building, Beijing \\ 100084, China \\ 'Pritzker School of Molecular Engineering, University of Chicago, 5640 South Ellis \\ Avenue, Chicago, Illinois 60637, United States \\ stephen.schrettl@unifr.ch, christoph.weder@unifr.ch
}

Received: 28.08 .2020

Accepted after revision: 07.10.2020

DOI: 10.1055/s-0040-1721052; Art ID: om-20-0028oa

License terms: COC

(c) 2020. The Author(s). This is an open access article published by Thieme under the terms of the Creative Commons Attribution-NonDerivative-NonCommercial-License, permitting copying and reproduction so long as the original work is given appropriate credit. Contents may not be used for commercial purposes, or adapted, remixed, transformed or built upon. (https://creativecommons.org/licenses/by-nc-nd/4.0/)

Abstract Mechanoresponsive luminescent (MRL) elastomers, which change their fluorescence color or intensity upon deformation, can facilitate simple strain detection through optical signals. Several polymers have been endowed with MRL properties by blending them with excimer-forming dyes, whose assembly and emission color are affected by deformation of the blended materials. However, access to elastic MRL polyurethanes based on this approach has proven difficult and usually requires the covalent incorporation of such dyes in high concentration. Here, we show that much simpler access to MRL elastomers is possible by blending thermoplastic polyurethane elastomers with a small weight fraction of a telechelic sensor macromolecule carrying two excimer-forming oligo( $p$-phenylene vinylene) dyes at the termini. While the mechanical properties of the two polyurethanes, which were selected because of their dissimilar mechanical behaviors, remain unchanged, the additive imparts these materials with MRL characteristics. Notably, the reliable and reversible detection of strains as low as $5 \%$ is possible. The highly sensitive mechanochromic response mirrors the deformation and relaxation processes occurring in these model polyurethanes and enabled a detailed analysis of the processes underlying the shape-memory properties in one of the polyurethanes, in which such behavior was imparted by a crystallizable soft segment.

Key words mechanochromic materials, stimuli-responsive materials, organic shape-memory polymers, aggregation

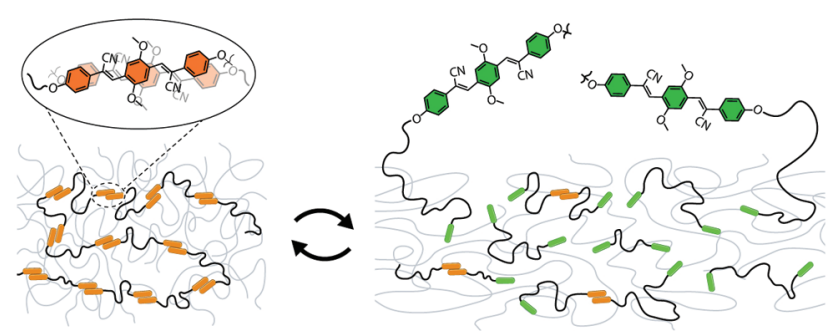

\section{Introduction}

Polymers that change their absorption and/or fluorescence color in response to mechanical deformation permit assessing the effects of mechanical forces, without the need for laborious analyses or expensive equipment. ${ }^{1}$ Such mechanochromic responses could be useful for the inspection of load-bearing structures, in which information about the location and extent of damage in the material is crucial, in particular to avoid catastrophic failure and enable the implementation of need-based servicing or replacement schemes. ${ }^{2}$ A popular strategy for fabricating mechanochromic polymer materials is the incorporation of so-called mechanophores, i.e., molecules featuring a covalent bond that breaks preferentially when subjected to excessive force. Frequently employed motifs include spiropyrans, 1,2-dioxetanes, and Diels-Alder adducts, and equipping polymers with these has allowed for the preparation of a variety of materials that display mechanochromic responses. ${ }^{3}$ For example, such mechanophores have been incorporated into polyurethanes that then display a color change upon tensile or compressive deformation. ${ }^{4}$ However, mechanophores typically require a high threshold force for activation, and the reverse reaction generally requires additional stimuli, which impedes the monitoring of relaxation processes. ${ }^{5}$

A particularly straightforward alternative means to create materials that display a mechanoresponsive luminescence (MRL) behavior involves the blending of excimer-forming dyes into a polymer matrix, or covalently integrating such dyes into a polymer backbone. ${ }^{1 e, 6}$ If the dyes are immiscible with the matrix, phase separation can lead to the formation of (ideally nanometer-sized) aggregates that fluoresce at a significantly longer wavelength than the well-solubilized dye molecules. When tensile deformation or compression disrupts a sufficiently large fraction of such aggregates, monomer emission is restored and this can lead to a 
perceptible fluorescence color change. ${ }^{6 a}$ Excimer-forming dyes that have been used in such simple blending approaches include oligo( $p$-phenylene vinylene) (OPV), ${ }^{6}$ perylene, ${ }^{7}$ poly (phenylene ethynylene), ${ }^{1 \mathrm{a}}$ and bis(benzoxazolyl)stilbene (BBS) derivatives. Polymer matrices that have been successfully rendered mechanochromic using this approach include poly(ethylene) (PE), ${ }^{1 \mathrm{a}, 1 \mathrm{~b}, 6 \mathrm{a}, 7,8}$ poly(ethylene terephthalate) (PET) ${ }^{9}$ poly(1,4-butylene succinate) ${ }^{10}$ poly(propylene) (PP), ${ }^{11}$ poly(vinylidene fluoride), ${ }^{12}$ and polyamide. ${ }^{13}$ This list reflects that the approach has largely been limited to semicrystalline polymers, and it appears that this microstructure and the significant local shear forces that it imparts are necessary to break up microscopic dye aggregates and elicit a notable fluorescence response. ${ }^{1 \mathrm{e}, 14}$ Moreover, semicrystalline matrices have been reported to nucleate the formation of dye aggregates and thereby influence their size in a considerable manner. ${ }^{8 \mathrm{~b}}$ By contrast, matrices with a high fraction of an amorphous phase and a low glass transition temperature promote the formation of large, hard-todissociate dye aggregates, on account of slow nucleation. ${ }^{6 \mathrm{~b}, 8 \mathrm{~b}}$ A notable exception is the use of BBS with a commercial, low-crystallinity polyurethane, in which the dye aggregates that form upon blending remain small enough to allow for reversible mechanochromism. ${ }^{15}$ However, the effectiveness of this strategy for fabricating mechanochromic materials has thus far been limited to this particular polyurethane/BBS combination, since the small aggregate size and the force transfer from the polymer host appear to be mediated by specific hydrogen-bonding interactions between the dye and the polymers' hard segments. ${ }^{15 a}$ Alternatively, covalent attachment of dyes to the polymer backbone has been shown to facilitate force transfer to the aggregates and limit their size, in turn eliciting a more significant force-dependent fluorescence response. ${ }^{6 \mathrm{~b}, 16}$ For example, a polyurethane containing OPV in the backbone displays a much greater mechanochromic response than the corresponding melt- or solutionprocessed blends with a free OPV dye. ${ }^{6 \mathrm{~b}}$ Despite the enhanced mechanochromic response, covalent dye incorporation significantly complicates the production of MRL materials and may not be straightforward for a wide range of polymer types and applications. Moreover, a much higher weight fraction of the dye is typically required in polymers that covalently integrate the dye, e.g., 5-20 wt\% for OPVs integrated in a polyurethane or a poly(ester), ${ }^{6 \mathrm{~b}, 16}$ whereas blends with semicrystalline PEs featuring only $0.1 \mathrm{wt} \%$ of an OPV dye display a mechanochromic response. ${ }^{8}$

To overcome this problem, some of us recently developed a telechelic poly(ethylene-co-butylene) carrying OPV dyes at the termini (tOPV) and demonstrated that blending this additive with different thermoplastic elastomers renders the latter mechanochromic(Figure 1a). ${ }^{17}$ The reversible response of such blends to mechanical deformation suggests that the MRL behavior may be mediated by entanglements between the macromolecular additive and the polymer matrix, rather than caused by the dissociation of dye aggregates through shear forces involving crystalline polymer domains. Here, we report that a reliable, sensitive, and reversible detection of mechanical stresses can also be achieved when tOPV is used as an additive in thermoplastic polyurethanes (TPUs), a class of materials that have been difficult to render mechanochromic via simple blending techniques. ${ }^{6 \mathrm{~b}}$ We investigated two TPU matrices that are structurally similar but display very different mechanical properties. Both polymers feature hard segments that are formed by the reaction of methylene diphenyl diisocyanate units and butanediol, which form physical cross-links. One of the polymers (referred to henceforth as PU) features a poly (tetrahydrofuran) soft segment, while the other (PBA-PU) contains poly(butylene adipate) (PBA) segments (Figure S1). The former phase-separates into largely amorphous domains that bestow PU with a low modulus and high elasticity, whereas the latter crystallizes, so that the deformation of PBAPU at ambient temperature is partially plastic. Furthermore, the additional thermal transition of the PBA domains imparts this material with shape-memory behavior. ${ }^{18}$ Monitoring the emission during uniaxial tensile deformation or relaxation of the TPU/tOPV blends allowed us to demonstrate that deformation reliably results in distinct, visually discernible fluorescence color changes in these different materials. Detailed characterization by in-situ optomechanical measurements in conjunction with stress-controlled shape-memory experiments shows that the fluorescence signal can be correlated with the polymer's response to mechanical deformation, further corroborating that the use of macromolecular aggregachromic additives enables an efficient force transduction. We anticipate that the results reported here will serve as a basis for expanding the use of such additives to a wide range of materials.

\section{Results and Discussion}

Blends of the commercially available TPUs PU and PBA-PU containing small amounts (0.2-1 wt\%) of the cyano-OPV functionalized telechelic poly(ethylene-co-butylene) (tOPV) additive (Figure 1a) were prepared by casting THF solutions of either PU or PBA-PU into poly(tetrafluoroethylene) (PTFE) molds before a solution of tOPV $\left[c(\mathrm{THF})=0.33 \mathrm{mmol} \mathrm{L}^{-1}\right]$ was added. After evaporation of the solvent, the resulting blends were compression-molded into films of a uniform thickness of 230-330 $\mu \mathrm{m}$ (see the Supporting Information for details). Thermogravimetric analyses of the blends show that the onset of mass loss is at temperatures $>300{ }^{\circ} \mathrm{C}$ (Figure S2) and the differential scanning calorimetry (DSC) heating and cooling traces match those of the neat polymers (Figure S3). The DSC traces of PU/tOPV feature only a glass transition $\left(T_{\mathrm{g}}\right)$ at ca. $-45^{\circ} \mathrm{C}$, confirming the amorphous nature of the phase formed by the poly(tetrahydrofuran) soft segments. In the case of PBA-PU/tOPV, the DSC traces display reversible transitions 

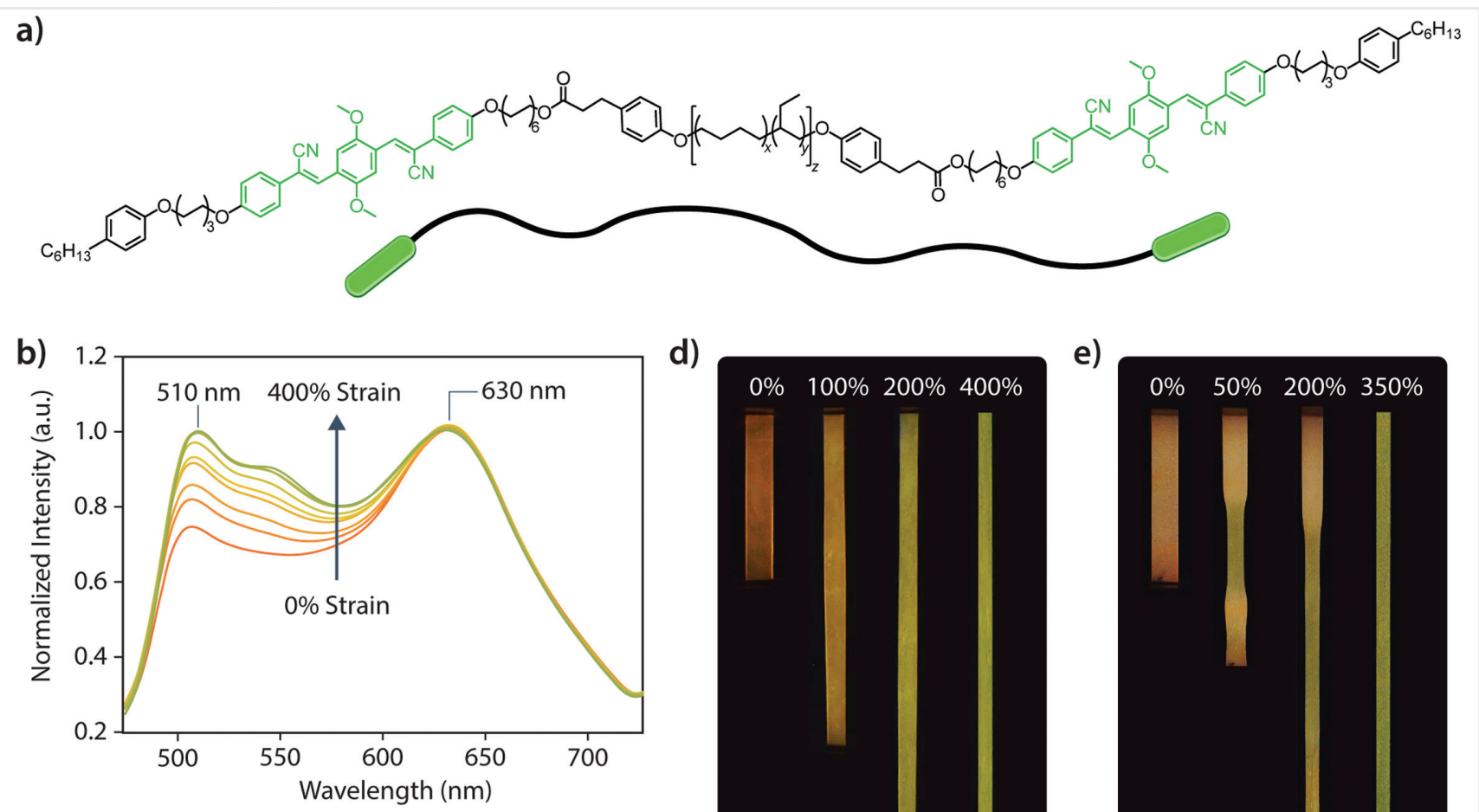

d)

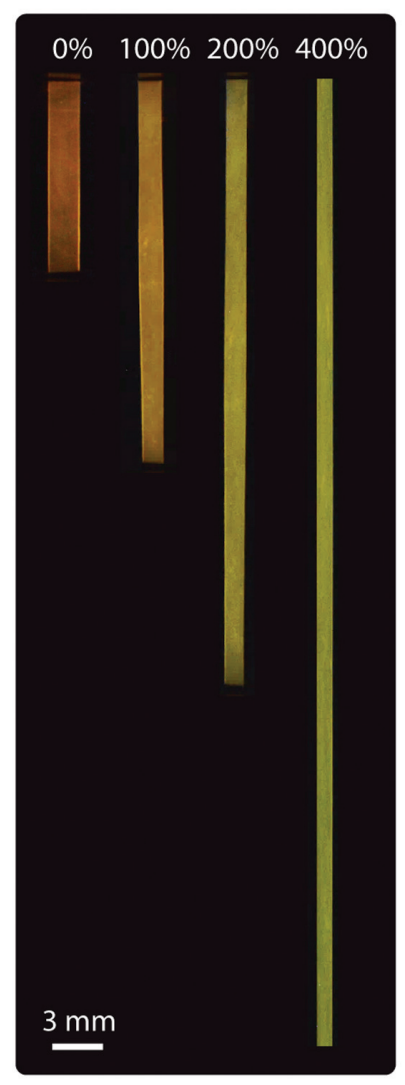

e)

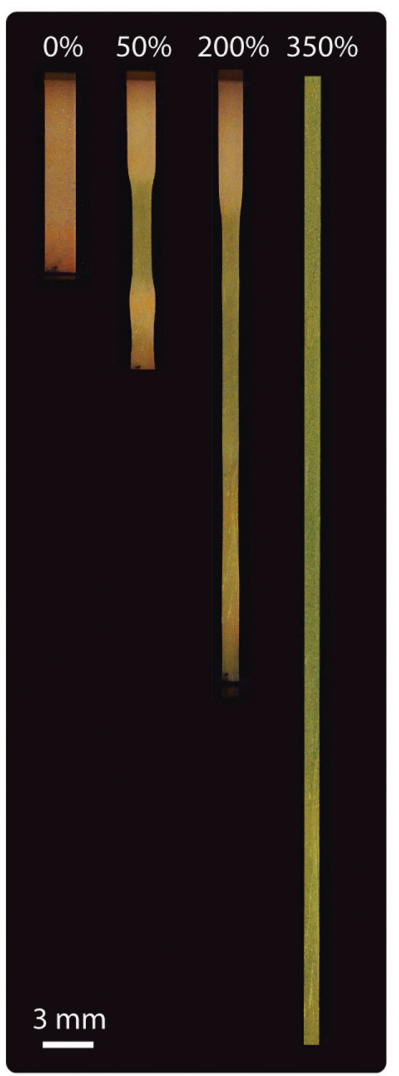

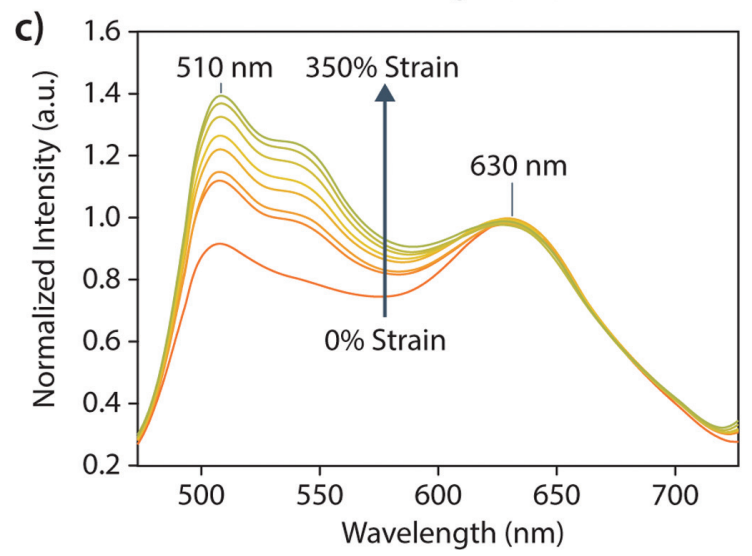

Figure 1 a) Chemical structure of the cyano-OPV functionalized telechelic poly(ethylene-co-butylene) (tOPV) that was employed as a strain-sensing additive for thermoplastic polyurethanes (with $M_{\mathrm{n}}=4500 \mathrm{~g} \mathrm{~mol}^{-1} ; x \approx 0.36, y \approx 0.64, z \approx 44$ ). b, c) Fluorescence spectra of films of (b) a PU/tOPV blend $(0.2 \mathrm{wt} \%)$ and (c) a PBA-PU/tOPV blend $(0.25 \mathrm{wt} \%)$ after being stretched to a strain of up to $400 \%$ in increments of $50 \%$; spectra were recorded after each strain increment and normalized to the excimer signal at $630 \mathrm{~nm}$. d, e) Images of samples of the (d) PU/tOPV (0.2 wt\%) and (e) PBA-PU/tOPV (0.25 wt\%) films taken under UV illumination $\left(\lambda_{\mathrm{ex}}=365 \mathrm{~nm}\right)$ after deformation to the indicated strains.

associated with the melting and crystallization of the PBA domains at ca. $50{ }^{\circ} \mathrm{C}$ and $-5{ }^{\circ} \mathrm{C}$, respectively. ${ }^{18 \mathrm{~h}, 19}$ The mechanical properties of the blends were probed by uniaxial tensile testing with dog-bone-shaped samples that were cut from the compression-molded films. The stress-strain curves recorded for PU/tOPV show the expected elastic behavior of a soft polyurethane elastomer with a Young's modulus of $19.8 \pm 0.3 \mathrm{MPa}$ (Figure S4). By contrast and on account of the crystalline PBA domains, PBA-PU/tOPV displays an elastic low-strain regime characterized by a Young's modulus of $118 \pm 6 \mathrm{MPa}$; the stress-strain curves show a yield point at ca.
$20 \%$ strain, beyond which plastic deformation is observed (Figures S4 and S5). A comparison of the stress-strain curves of the neat polymers and the blends shows no significant differences, confirming that the addition of small quantities of the tOPV additive via simple blending does not alter the properties.

When placed under UV illumination, the PU and PBA-PU blends with as little as $0.2-0.25 \mathrm{wt} \%$ of the tOPV additive (equivalent to $0.04-0.05$ wt\% of the dye) display a homogenous yellow-orange fluorescence, whereas the neat polymers weakly fluoresce blue (Figures S6 and S7). As previously 
reported, ${ }^{17}$ the neat tOPV additive shows green emission in dilute solution $\left[c\left(\mathrm{CHCl}_{3}\right)=1.4 \mu \mathrm{mol} \mathrm{L}^{-1}\right]$ with a maximum of $\lambda_{\max }=505 \mathrm{~nm}$, while orange excimer luminescence characterized by a band centered at $634 \mathrm{~nm}$ is observed after complete evaporation of the solvent. Fluorescence spectra of the PU/tOPV and PBA-PU/tOPV blends investigated here show the corresponding bands for the monomer and excimer with maxima at 510 and $630 \mathrm{~nm}$, respectively (Figures 1b, c, and S8). The ratio of monomer-to-excimer emission $\left(I_{510} / I_{630}\right)$ was used as a metric to monitor the extent of aggregation. In both blend series, the $I_{510} / I_{630}$ ratio decreases as the concentration of the tOPV additive is increased, reflecting a larger fraction of dye aggregates. A comparison of the spectra of solvent-cast and compression-molded samples indicates that the latter display a slightly higher $I_{510} / I_{630}$ ratio (Figure S9), suggesting kinetic trapping of some of the tOPV molecules upon cooling from the melt. Different cooling rates between 1 and $10^{\circ} \mathrm{C} \mathrm{min}^{-1}$ were found to furnish samples that exhibit the same $I_{510} / I_{630}$ ratio. Emission spectra acquired during heating of a PBA-PU/tOPV $(0.25 \mathrm{wt} \%)$ blend show that the relative contribution of excimer emission drops upon heating until, at temperatures above $120^{\circ} \mathrm{C}$, only monomer emission is observed (Figure S10a). DSC measurements show that neat tOPV undergoes a melting transition between 108 and $129^{\circ} \mathrm{C}$ (Figure S11), which is in line with the previously reported values for this additive. ${ }^{17}$ Monitoring PU/tOPV and PBA-PU/tOPV blend films upon heating to $120{ }^{\circ} \mathrm{C}$ $\left(10{ }^{\circ} \mathrm{C} \mathrm{min}{ }^{-1}\right)$ reveals that the $I_{510} / I_{630}$ ratio increases above ca. $60{ }^{\circ} \mathrm{C}$, and this increase is expedited above ca. $100{ }^{\circ} \mathrm{C}$ (Figure S10b, c), suggesting that tOPV micro-phase-separates from the TPUs. The $I_{510} / I_{630}$ ratio was only partially recovered upon cooling, presumably due to the photoinduced isomerizations OPV dyes reportedly undergo when continuously irradiated. $^{20}$ However, heating a PBA-PU/tOPV (0.25 wt\%) sample to $120{ }^{\circ} \mathrm{C}$ and acquiring spectra at temperature increments of $20^{\circ} \mathrm{C}$ at different spots of the sample served to demonstrate that the $I_{510} / I_{630}$ ratio displays the same trend, while the emission intensity ratio is almost completely recovered after cooling to $20{ }^{\circ} \mathrm{C}$ (Figure S10d). The $I_{510} / I_{630}$ ratio of samples that were afterwards kept at $20^{\circ} \mathrm{C}$ continued to decrease over the course of $1 \mathrm{~h}$, indicating that a further reaggregation of a small amount of the tOPV additive occurs slowly. To ensure a uniform behavior of samples and an equilibrium aggregation state of the tOPV additive, all compression-molded samples were left at room temperature for at least $3 \mathrm{~h}$ prior to further testing.

To investigate the MRL response, compression-molded films of the PU blends with $0.2-1.0 \mathrm{wt} \%$ of tOPV were cut into rectangular strips $(30 \times 3 \mathrm{~mm})$ and subjected to uniaxial tensile deformation in a mechanical stretching frame that allowed recording fluorescence spectra at various strains (see the Supporting Information for details). The spectra of PU/tOPV (0.2 wt\%) samples show an increase of the monomer fluorescence upon deformation, indicating disaggregation of the chromophores (Figure 1b). This effect also manifests itself in a visually discernible change of the emission color from orange to green when samples are excited with UV light (Figure 1d, Video $1 ; \lambda_{\mathrm{ex}}=365 \mathrm{~nm}$ ). The $I_{510} / I_{630}$ ratio of samples with varying tOPV concentrations (0.2-0.5 wt\%) increases in all cases almost linearly with the strain (Figure S12). The uniaxial deformation of films of the semicrystalline PBA-PU/tOPV ( $0.25 \mathrm{wt} \%$ ) blend beyond the yield point leads to significant necking that starts at the center of the film and propagates outwards (Figure 1e). An increase in monomer emission can be seen in the parts of the sample that have undergone plastic deformation and a concomitant, visually discernible irreversible change of the emission color from orange to green is observed (Figure 1c, e; Video 2; $\lambda_{\mathrm{ex}}=365 \mathrm{~nm}$ ). The monomer emission continues to increase until the material fluoresces green at a strain of ca. $350 \%$ (Figure 1e). PBA-PU/tOPV blends that feature different concentrations of the tOPV additive (0.25-1.0 wt\%) all display similar MRL behavior and plots of the $I_{510} / I_{630}$ ratio against the applied strain show that the curves are roughly parallel to each other (Figure S12). Compositions with a tOPV concentration of between $0.2 \mathrm{wt} \%$ (PU/tOPV) and $0.25 \mathrm{wt} \%$ (PBA-PU/tOPV) display the most striking fluorescence color change upon deformation, since the dominant emission color of the pristine blend films is orange, while green emission dominates in the deformed samples; therefore, these additive concentrations were used for most of the subsequent experiments. For compositions with a higher tOPV content, the changes can be readily monitored spectroscopically, but the change in the dominant emission is not observed (Figures S12 and S13). Taken together, these data show that simple blending with low concentrations of the macromolecular tOPV additive is an effective means to render both amorphous and semicrystalline PU mechanochromic, and the change in the material's emission during mechanical deformation can be monitored spectroscopically and is visually detectable under UV illumination for blends of a suitable composition.

To demonstrate that the MRL response achieved with the telechelic tOPV additive in the two selected TPUs cannot be achieved with low-molecular-weight aggregachromic dyes, blends of PU with the OPV derivative 1,4-bis( $\alpha$-cyano-4octadecyloxystyryl)-2,5-dimethoxybenzene (C18-RG) or 4,4'-bis(2-benzoxazolyl)stilbene (BBS) were prepared, since these dyes were previously used very successfully with a range of different polymer matrices, including PET, ${ }^{9}$ polyamide- $12,{ }^{13} \mathrm{PP}^{11}$ and some PU polymers. ${ }^{6 \mathrm{~b}, 15}$ Films of PU/BBS blends (0.05-0.5 wt\%) only display negligible fluorescence changes upon uniaxial deformation up to $400 \%$ strain (Figures S14 and S15), while PU/C18-RG blends (0.2-2.0 wt\%) showed significant macro- and microscopic phase separation (Figures S16 and S17). Blending C18-RG with PBA-PU furnished macroscopically homogeneous films 
(0.6 wt\% C18-RG; Figure S17d), but no change in fluorescence was observed upon uniaxial tensile deformation (Figure S17). This comparison unambiguously demonstrates that the limitations of phase separation and poor responses in elastic matrices that are typically encountered with blends of aggregachromic dyes can be readily overcome through the use of the tOPV additive.

In order to demonstrate the utility of the tOPV additive as a means to monitor mechanical deformation, additional in-situ fluorescence measurements were performed during uniaxial tensile deformation, stress-relaxation, and cyclic loadingunloading experiments. To this end, an optical fiber connected to a spectrometer was aligned with the center of samples that were mounted in a tensile tester and emission spectra were acquired at a rate of $0.33 \mathrm{~Hz}$ during and, optionally, after deformation. After screening strain rates from $5 \% \mathrm{~min}^{-1}$ to $300 \% \min ^{-1}$ with samples of PBA-PU (Figure S18), an intermediate strain rate of $50 \% \mathrm{~min}^{-1}$ was chosen for all subsequent experiments. At lower strain rates, the deforma- tion was less homogeneous, whereas only a limited number of fluorescence measurements can be acquired at higher strain rates. A comparison of the stress-strain curves of PU/tOPV (0.2-0.5 wt\%) and PBA-PU/tOPV (0.25 wt\%) blend films and the in-situ measured $I_{510} / I_{630}$ values shows that the mechanochromic response of the two systems varies considerably, indicative of different deformation processes in the two TPUs. In the case of the elastic PU/tOPV blends, the changes of the $I_{510} /$ $I_{630}$ ratio correlate well with the applied strain (Figures $2 \mathrm{a}$ and S19a, b). Interestingly, two distinct linear regimes $\left(R^{2} \geq 0.92\right)$ are discernible in the $I_{510} / I_{630}$ vs. strain plot. The transition between these regimes at a strain of ca. $130 \%$ coincides with an inflection point in the stress-strain curve that marks the onset of the strain-hardening region, suggesting that the $I_{510} / I_{630}$ ratio reflects the changing viscoelastic response of the PU matrix. In the case of the PBA-PU/tOPV blends, a pronounced change in the $I_{510} / I_{630}$ ratio only occurs after deformation beyond the yield point (Figures $2 \mathrm{~b}$ and S19c, d), i.e., in the plastic deformation regime. Thus, a
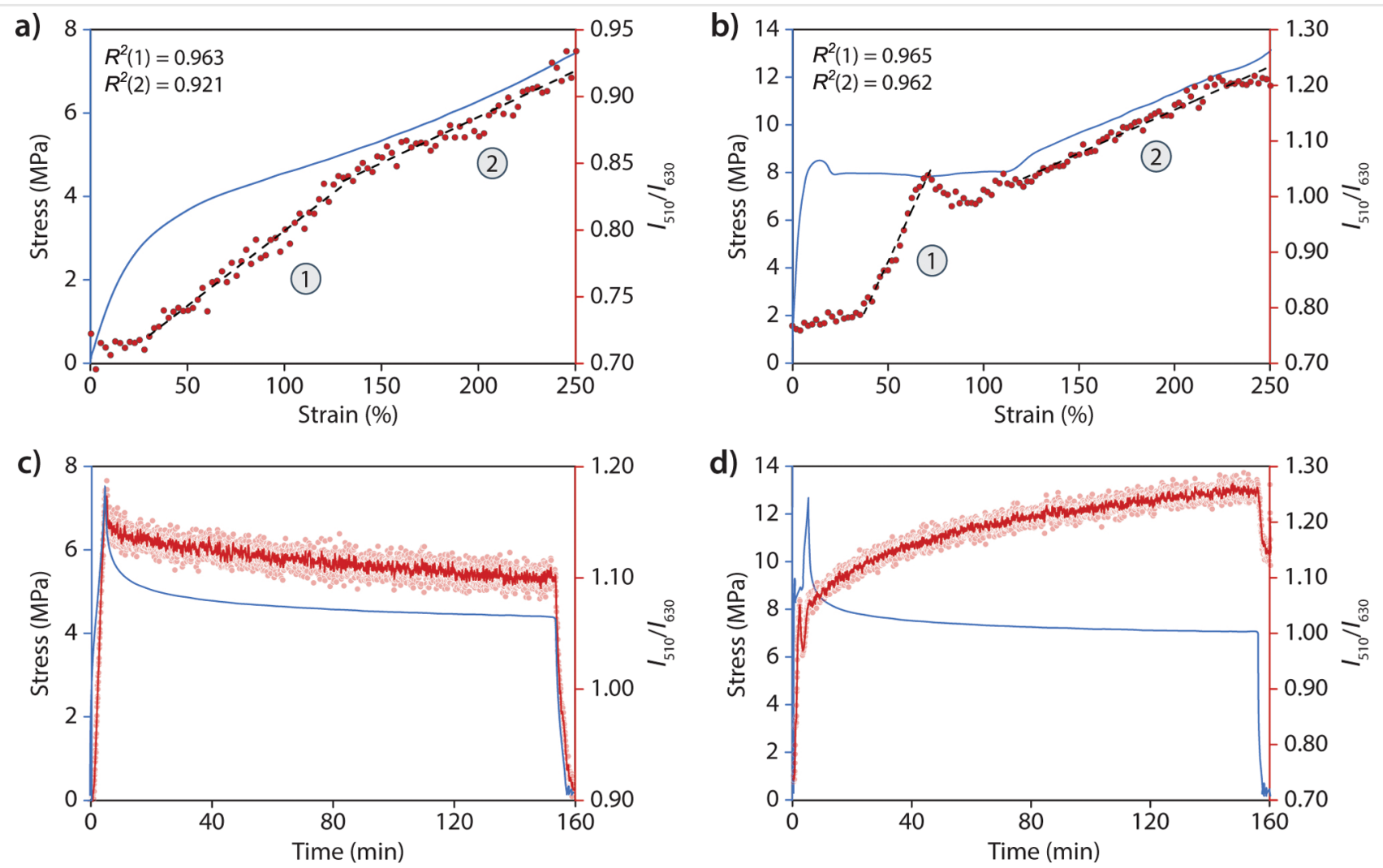

Figure 2 Fluorescence color change of PU/tOPV (0.2 wt\%) and PBA-PU/tOPV (0.25 wt\%) blend films during (a, b) uniaxial tensile deformation and (c, d) equilibration under continuously applied strain. a, b) The comparison of the stress-strain curves (blue lines) and the monomer-to-excimer intensity ratio $\left(I_{510} / I_{630}\right.$, red dots) as a function of strain shows a correlation between the strain and the emission characteristics. Linear fits of the $I_{510} / I_{630}$ data appear to indicate different extension regimes (1,2; represented by black dashed lines). Blends containing tOPV at other concentrations show a similar response (Figure S19). c, d) Plots of the $I_{510} / l_{630}$ ratio (red dots; dark red lines represent a smoothened 5-point average) and stress (blue lines) recorded during extension of films to a strain of ca. $250 \%$, subsequent equilibration under a fixed strain over the course of $2.5 \mathrm{~h}$, and finally release of the stress and relaxation of the samples. The evolution of $I_{510} / I_{630}$ in the holding segments is similar for blend films containing different concentrations of tOPV (Figure S20). In all experiments a strain rate of $50 \% \mathrm{~min}^{-1}$ was applied. 
steep increase in $I_{510} / I_{630}$ is observed at a strain of ca. $15-50 \%$; due to the experimental setup, the value varies from sample to sample and depends on where the neck forms and when it propagates through the center of the sample where fluorescence is monitored. Further deformation leads to a moderate increase of the $I_{510} / I_{630}$ ratio that linearly correlates $\left(R^{2}=0.97\right)$ with the applied strain and follows a slope that is comparable to the one observed for PU/tOPV in the strain-hardening region.

The different responses of the two matrix polymers upon mechanical deformation are also apparent in stressrelaxation experiments. When films of the elastic PU/tOPV blend ( $0.2 \mathrm{wt} \%$ ) were deformed to and maintained at $250 \%$ strain, stress relaxation occurs over the course of ca. $60 \mathrm{~min}$ (Figures $2 \mathrm{c}$ and S20a). The $I_{510} / I_{630}$ ratio mirrors this behavior, showing a steady decrease of the monomer emission over time that follows the reduction in stress and approaches a plateau (Figure 2c), likely as a consequence of chain relaxations that facilitate re-aggregation of a fraction of the tOPV additives. The same experiment was also carried out with PBA-PU/tOPV (0.25 wt\%) blends. In this case a plot of the $I_{510} / I_{630}$ ratio shows that the tOPV monomer signal increases during the stress-relaxation step in a logarithmic manner (Figures 2d and S20-S22), even though the stress relaxation is similar to that of the PU/tOPV blends. The increase of the monomer emission when holding samples of the PBA-PU blend under tension is possibly caused by local strains that are related to strain-induced crystallization of the PBA, a phenomenon that is known to occur when polyurethane elastomers containing polyester or polyether soft segments are placed under tensile stress. ${ }^{18 \mathrm{e}, 21}$ Indeed, samples of PBA-PU/tOPV (0.25 wt\%) that were deformed to a strain of $300 \%$ display a pronounced melting transition in the first DSC heating trace (Figure S23), while the DSC trace of a PU/tOPV (0.25 wt\%) film that was repeatedly deformed to $300 \%$ strain mirrors the one of a pristine sample. Sample fluorescence was also monitored while extending films to $250 \%$ strain and subsequently releasing them from the clamps of the tensile tester. To directly compare the fluorescence changes, PU/tOPV and PBA-PU/tOPV blends with $0.25 \mathrm{wt} \%$ of the additive were used. In the case of PU/tOPV (0.25 wt\%), only a slight initial increase of the monomer emission was observed that can be ascribed to photoinduced reactions of the OPV dyes (Figure S21), ${ }^{20}$ particularly since this increase is also observed for pristine samples (Figure S22). By contrast, the same experiment with a PBA-PU/tOPV (0.25 wt\%) blend film shows that sample relaxation is followed by a logarithmic increase in the $I_{510} / I_{630}$ ratio over the course of $1.5 \mathrm{~h}$ (Figures S20 and S21). The fluorescence response reflects that relaxation processes continue to occur in plastically deformed samples of the semicrystalline PBA-PU. Indeed, extension and stressrelaxation experiments with PBA-PU/tOPV (0.25 wt\%) samples carried out at $55{ }^{\circ} \mathrm{C}$, i.e., above the melting transition of the PBA domains, show that the mechanical properties and fluorescence response closely resemble the behavior of PU/tOPV blends (Figure S24).

To further demonstrate the utility of the tOPV additive in enabling a reversible mechanochromic response in these different polyurethanes, cyclic loading-unloading experiments with both blends were carried out. The results show that (dis)aggregation of the additive is fully (PU/tOPV) or partially (PBA-PU/tOPV) reversible over at least five deformation and relaxation cycles (Figures 3 and S25-S27), mirroring the behavior of the respective polymer matrices in their corresponding stress-strain curves (Figure S28). To facilitate the detection of minor changes in the $I_{510} / I_{630}$ ratio during cyclic deformation, PU/tOPV blends with $0.5 \mathrm{wt} \%$ were used. Following the changes in the $I_{510} / I_{630}$ ratio of these samples upon extension to a strain of $250 \%$, the original fluorescence color and $I_{510} / I_{630}$ ratio are almost completely recovered after relaxation (Figure 3a). Interestingly, in subsequent extension and contraction cycles, the $I_{510} / I_{630}$ ratio follows the strain even more closely than in the first deformation cycle (Figures 3a and S25-S26). A detailed analysis of in-situ fluorescence spectroscopy data recorded during deformation of pristine $\mathrm{PU} / \mathrm{tOPV}(0.5 \mathrm{wt} \%)$ to a maximum strain of only $30 \%$ shows that a reversible mechanochromic response is observed before significant plastic deformation occurs (Figures $3 c$ and S29). The increase of the $I_{510} / I_{630}$ ratio can be detected at strains at least as low as $5 \%$ (Figures $3 d$ and S29), suggesting an efficient transfer of the small mechanical stresses of the surrounding matrix to the tOPV aggregates.

The results of cyclic loading-unloading experiments with films of the PBA-PU/tOPV (0.25 wt\%) blend show that the first deformation of a pristine sample to a strain of $300 \%$ causes significant plastic deformation (Figure $3 \mathrm{~b}$ ), with a recoverable strain of ca. 100\% (Figures 3b, S28c, and Video 2). The limited recovery is clearly reflected by the $I_{510} / I_{630}$ ratio, which increases from 0.8 (as-prepared film) to 1.1 after the first loading and unloading cycle. In subsequent deformation cycles, the sample fully recovers, and the $I_{510} / I_{630}$ ratio traces the applied stress and strain reasonably well, although a "drift" towards monomer emission is evident. Indeed, the increase in monomer emission observed for the PBA-PU/tOPV films in stress-relaxation experiments (Figure $2 \mathrm{~d}$ ) is reflected in the increasing baseline of the $I_{510} / I_{630}$ ratio after unloading to 0.1 MPa (Figures 3b and S27). The in-situ optomechanical characterization of the PU/tOPV and PBA-PU/tOPV blends unambiguously demonstrates that the use of tOPV as an additive imparts MRL behavior to very different types of polymer matrices. Moreover, the observed response at relatively low strains in an elastomer strongly supports that chain entanglement between the matrix and the additive's poly(ethylene-co-butylene) backbone is what enables the efficient transfer of mechanical stresses, ${ }^{17}$ and the precise mechanism of force transfer is currently under investigation.

To further demonstrate the usefulness of the tOPV additive to monitor complex thermomechanical processes, 

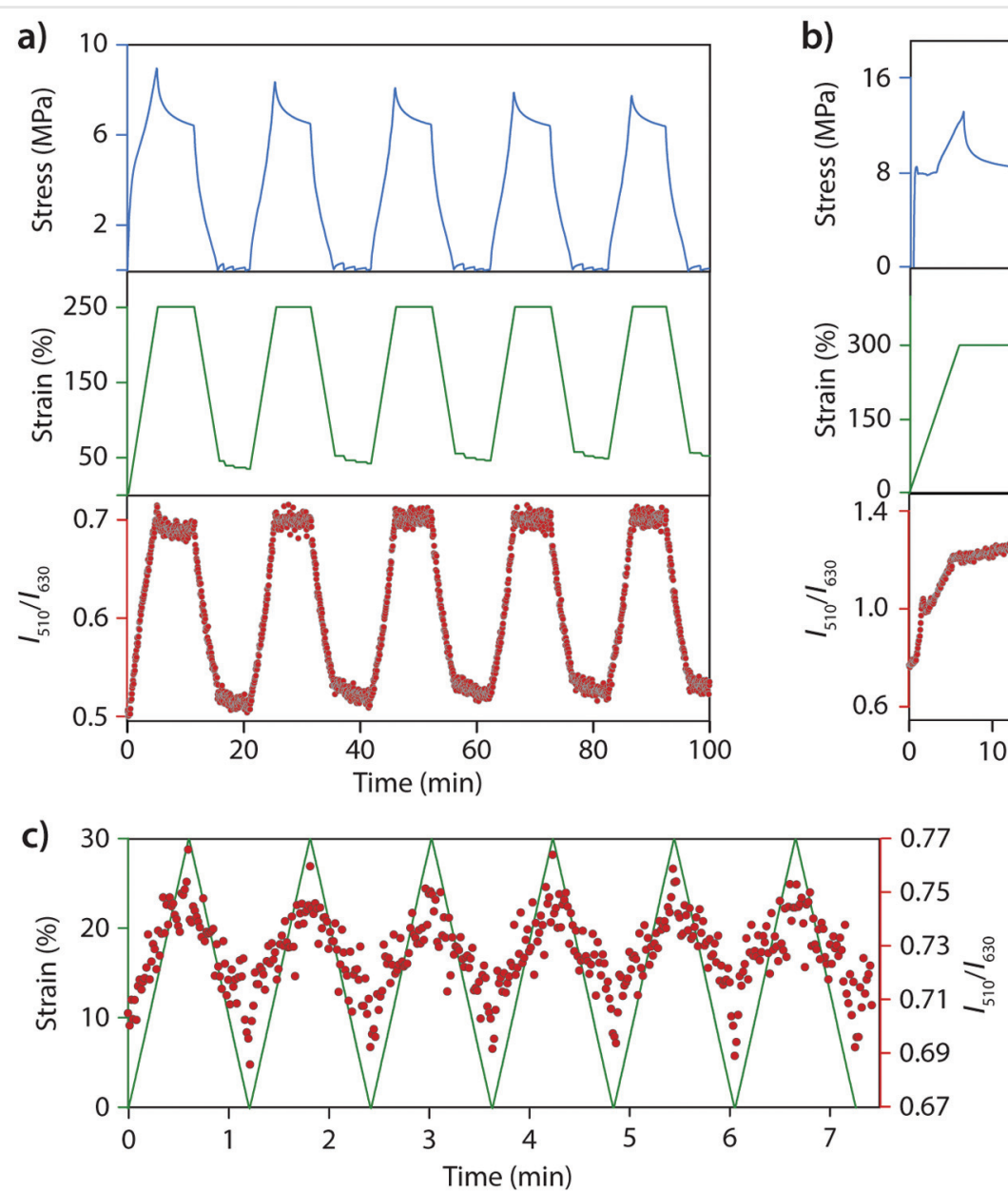
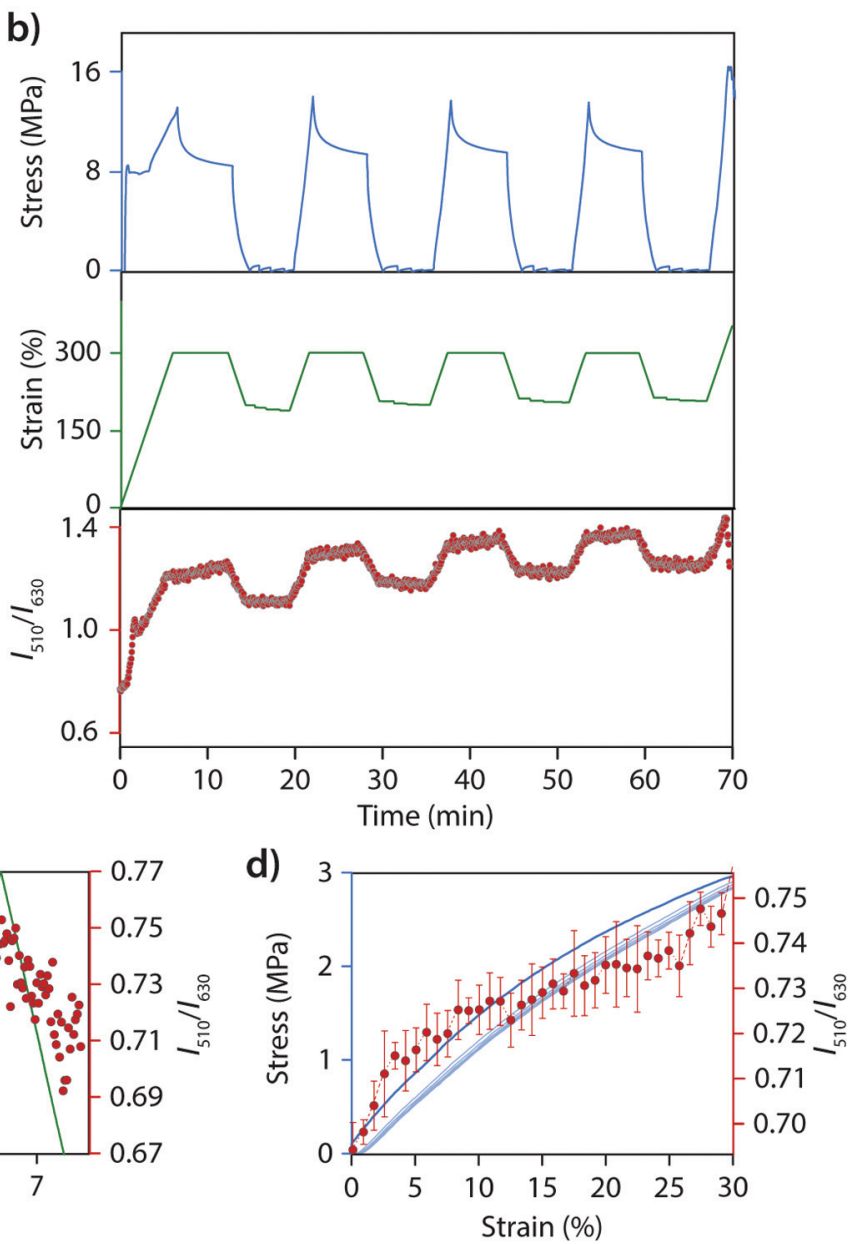

Figure 3 Plots showing the results of cyclic deformation experiments of (a, c, and d) PU/tOPV (0.5 wt\%) and (b) PBA-PU/tOPV (0.25 wt\%) blends. Shown are the time-stress (blue) and time-strain (green) curves and the corresponding fluorescence response $\left(I_{510} / I_{630}\right.$ ratio, red points). Experiments were conducted by controlling the strain (a: $250 \%$, b: $300 \%$, c, d: $30 \%$ ). d) The comparison of the stress-strain curves (blue lines) and the $I_{510} / I_{630}$ ratio (each red dot represents the average of data points from all six cycles; error bars correspond to one standard deviation) as a function of strain recorded during each extension cycle of (c) shows a very good correlation.

shape-memory programming and release experiments were carried out with films of the semicrystalline PBA$\mathrm{PU} / \mathrm{tOPV}(0.25 \mathrm{wt} \%)$ blend while simultaneously monitoring the fluorescence response. Thus, films were heated to $55^{\circ} \mathrm{C}$ (i.e., above the $T_{\mathrm{m}}$ of the PBA domains) and uniaxially deformed at this temperature (using stress control; see the Supporting Information for details) to a strain of $45 \%$ (Figures 4, S30, and Video 3). The temporary shape was then fixed by keeping the stress constant $(0.9 \mathrm{MPa})$, while the sample was cooled to $0{ }^{\circ} \mathrm{C}$ and maintained at this temperature for $5 \mathrm{~min}$. Due to the crystallization of the PBA segments in the fixing step, the sample further elongated to a strain of ca. $70 \%$. The sample showed little contraction ( $1.5 \%$ strain) when the stress was reduced to the lowest possible level (a preload of $0.15 \mathrm{MPa}$ ), indicating good fixity of the temporary shape (98\%). Finally, the recovery of the permanent shape was triggered by heating the sample again to $55{ }^{\circ} \mathrm{C}$ while keeping the stress at a minimum level $(0.15 \mathrm{MPa})$. Gratifyingly, the original shape was practically fully recovered, with a recovery ratio of $90 \%$ for the first cycle and 100\% for all subsequent cycles. This cycle was repeated three times (Figure S30) and the fluorescence response was spectroscopically monitored during the last cycle. Thus, when PBA-PU/tOPV (0.25 wt\%) was heated to $55{ }^{\circ} \mathrm{C}$ and deformed to $45 \%$ strain, the $I_{510} / I_{630}$ ratio was found to correlate with the applied strain (Figure 4). During shape fixing, the PBA-PU/tOPV sample is held under tension while being cooled to $0{ }^{\circ} \mathrm{C}$, which induces PBA crystallization and leads to a concomitant elongation and increase in the $I_{510} / I_{630}$ ratio. One may speculate that the increased monomer emission is caused by internal strains that arise when the crystallization of PBA domains leads to a local contraction, in line with the abovediscussed observations during stress relaxation at room 

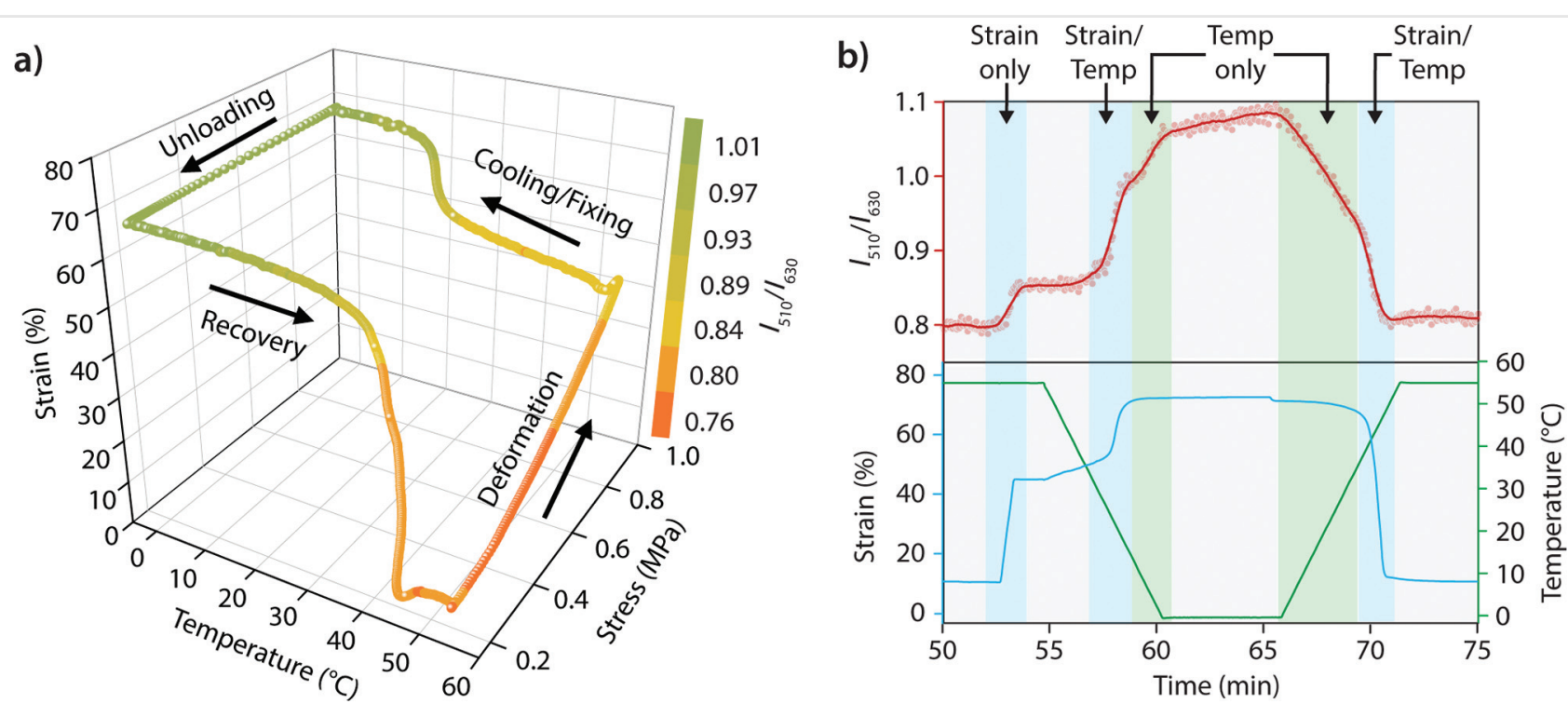

Figure 4 Shape-memory cycle carried out on a PBA-PU/tOPV ( $0.25 \mathrm{wt} \%$ ) blend film while monitoring fluorescence in situ. a) The fluorescence response was followed via the $I_{510} / I_{630}$ ratio, which is represented using the displayed color code, during the last of three consecutive shape-memory cycles. The minor signal fluctuations that occur at the end of the recovery and deformation phases are due to instrument artifacts, stemming from the fact that the tensile equipment used is not optimized for stress-controlled shape-memory cycling. b) Two-dimensional representation of the data in part (a). The lower panel plots the strain (blue line) and temperature (green line) profiles applied during the shape-memory cycle. The upper panel plots the corresponding fluorescence response (red dots; dark red line represents a smoothened 20-point average).

temperature (Figures 2d and S20-S23). While no significant changes to the $I_{510} / I_{630}$ ratio were observed when samples were unloaded in the programmed shape, melting the PBA domains by heating to $55^{\circ} \mathrm{C}$ and subsequent release of the strain for shape recovery led to the reformation of dye aggregates and a recovery of the $I_{510} / I_{630}$ ratio. Thus, the fluorescence response shows that the tOPV additive provides a reliable way to directly monitor the complex, internal stresses occurring in PBA-PU during a shapememory cycle.

\section{Conclusions}

In summary, we have shown that blending TPU elastomers with a small weight fraction of a telechelic additive carrying two excimer-forming OPV dyes at the termini provides straightforward access to MRL elastomers. The fluorescence response due to dissociation of dye aggregates is highly sensitive to the deformation and relaxation processes that occur in the herein investigated different types of polymer matrices. A detailed analysis of the MRL behavior shows that the application of low strains already furnishes an easily detectable and reliable mechanochromic response, regardless of the crystallinity of the host matrix. The fluorescence response was found to scale with the applied strain and its release during multiple cycles of deformation or relaxation, respectively. Moreover, complex processes upon stress relaxation under tension or during a shape-memory cycle were readily followed by monitoring the fluorescence changes. To achieve a defined MRL response with low-molecular-weight aggregachromic dyes, the latter generally need to be tailored to a given host polymer or covalently integrated to avoid excessive phase segregation and the formation of bulky, persistent aggregates. Given that tOPV fluorescence behavior appears to be intricately linked to the mechanically induced rearrangements of the surrounding matrix, such additives should be able to provide information about the nature and extent of (very small) deformations experienced by a priori any elastomeric material. We anticipate that similar mechanochromic additives bearing different excimer-forming dyes at the termini or comprising different cores may further expand the tunability of such deformation sensors.

\section{Experimental Section}

The telechelic tOPV additive and C18-RG dye used in this study were prepared as previously reported. ${ }^{9,17}$ BBS (97\%) was obtained from Sigma Aldrich and used without further purification. The TPU elastomers PBA-PU (Desmopan 2795A; number-average molecular weight $M_{\mathrm{n}}=93 \mathrm{kDa}$, $Ð=2.1$ ) and PU (Texin 985; $M_{\mathrm{n}}=111 \mathrm{kDa}, \emptyset=2.0$ ) were received in the pellet form from Covestro AG and were used as received. The polyurethane/tOPV blends were prepared by solvent casting the polymer and tOPV from THF solutions into a PTFE mold with a diameter of $6 \mathrm{~cm}$, drying overnight, 
and subsequently compression-molding the materials at $170-180{ }^{\circ} \mathrm{C}$ to obtain $230-330 \mu \mathrm{m}$ thick films. Blend films containing C18-RG and BBS dyes were fabricated following the same process. Films were deformed in a stepwise manner using a custom-made stretching frame, stressstrain curves were measured on a Zwick/Roell Z010 tensile testing machine with a $200 \mathrm{~N}$ load cell, and mechanical tests with in-situ fluorescence measurements were carried out using a Linkam TST350 microtensile stage equipped with a $20 \mathrm{~N}$ load cell. Unless otherwise noted, a strain rate of $50 \%$ $\min ^{-1}$ was used. Films for mechanical testing were prepared either as rectangular films (with dimensions of $3.0 \times 30 \mathrm{~mm}$ for manual stretcher and microtensile experiments) or as ASTM standard dog-bone-shaped samples (with dimensions of $5.0 \times 40 \mathrm{~mm}$ for experiments conducted on the Zwick/Roell Z010 tensile tester). In-situ fluorescence measurements were performed using an Ocean Optics USB 4000 spectrometer connected to an Ocean Optics LS-450 LED light source with an excitation wavelength of $\lambda_{\mathrm{ex}}=380 \mathrm{~nm}$ and an Ocean Optics QR230-7XSR SMA 905 optical fiber positioned perpendicular to the film at a distance of approximately $2 \mathrm{~mm}$. Fluorescence microscopy images were acquired in reflectance mode using an Olympus BX51 microscope with an integrated Olympus DP72 camera. Photographs of as-prepared and stretched films were taken with a Nikon D7100 digital camera equipped with an AF-S DX Zoom-NIKKOR 18-135mm lens (f/3.5-5.6G IF-ED).

\section{Video 1}

Reversible deformation of a PU/tOPV (0.2 wt\%) sample. (Repeated extension and contraction cycles recorded under UV light illumination (365 nm).) Online content including video sequences viewable at: https://www. thieme-connect.com/products/ejournals/html/ 10.1055/s-0040-1721052.

\section{Video 2}

Deformation of a PBA-PU/tOPV (0.25 wt\%) sample. (Manual sample deformation recorded under UV light illumination (365 nm).) Online content including video sequences viewable at: https://www. thieme-connect.com/products/ejournals/html/ 10.1055/s-0040-1721052.

\section{Video 3}

Shape memory cycle with a sample of PBA-PU/tOPV (0.25 wt\%). (Movie recorded under UV light illumination (365 nm) during the shape memory cycle shown in Fig. 4.) Online content including video sequences viewable at: https://www. thieme-connect.com/products/ejournals/html/ 10.1055/s-0040-1721052.

\section{Funding Information}

The authors gratefully acknowledge financial support through the National Center of Competence in Research (NCCR) Bio-Inspired Materials, a research instrument of the Swiss National Science Foundation (SNF), as well as funding from the Adolphe Merkle Foundation.

\section{Acknowledgments}

The authors would like to thank Veronique Buclin for her assistance in synthesizing the telechelic OPV reported in this work, Dr. Feyza Karasu Kilic for help with the Desmopan shape-memory experiments, Felipe Saenz for advice relating to photophysical characterization of the dye blend films, Livius F. Muff for his technical assistance in setting up the in-situ optomechanical microtensile experiments, and Cedric Kilchoer for help with solid-state optical measurements.

\section{Supporting Information}

Supporting information for this article is available online at https://doi.org/10.1055/s-0040-1721052.

\section{Primary Data}

The primary data generated during this study are available at: https://doi.org/10.5281/zenodo.4249552.

\section{References}

(1) (a) Pucci, A.; Biver, T.; Ruggeri, G.; Meza, L. I.; Pang, Y.Polymer 2005, 46, 11198. (b) Pucci, A.; Ruggeri, G. J. Mater. Chem. 2011, 21, 8282. (c) Weder, C. Mechanochromic Polymers. In: Encyclopedia of Polymeric Nanomaterials. Spinger: Berlin/Heidelberg; 2013,1-11. (d) Haehnel, A. P.; Sagara, Y.; Simon, Y. C.; Weder, C. Top. Curr. Chem. 
2015, 369, 345. (e) Calvino, C.; Neumann, L.; Weder, C.; Schrettl, S.J. Polym. Sci. Part A: Polym. Chem. 2017, 55, 640. (f) Kempe, F.; Brügner, O.; Buchheit, H.; Momm, S. N.; Riehle, F.; Hameury, S.; Walter, M.; Sommer, M. Angew. Chem. Int. Ed. 2018, 130, 1009. (g) Pucci, A. Sensors 2019, 19, 4969. (h) Zessin, J.; Schnepf, M.; Oertel, U.; Beryozkina, T.; König, T. A. F.; Fery, A.; Mertig, M.; Kiriy, A. Adv. Opt. Mater. 2020, 8, 1901410.

(2) (a) Kessler, S. S.; Spearing, S. M.; Soutis, C. Smart Mater. Struct. 2002, 11, 269. (b) Cawley, P. Struct. Health Monit. 2018, 17, 1225.

(3) (a) Li, J.; Nagamani, C.; Moore, J. S. Acc. Chem. Res. 2015, 48, 2181. (b) Stratigaki, M.; Göstl, R. ChemPlusChem 2020, 85, 1095. (c) Deneke, N.; Rencheck, M. L.; Davis, C. S. Soft Matter 2020, 16, 6230. (d) Binder, W. H. Polymer 2020, 202, 122639.

(4) (a) Lee, C. K.; Davis, D. A.; White, S. R.; Moore, J. S.; Sottos, N. R.; Braun, P. V.J. Am. Chem. Soc. 2010, 132, 16107. (b) Verstraeten, F.; Göstl, R.; Sijbesma, R. P. Chem. Commun. 2016, 52, 8608. (c) Yuan, W.; Yuan, Y.; Yang, F.; Wu, M.; Chen, Y. Macromolecules 2018, 51, 9019.

(5) Cao, Z. Macromol. Chem. Phys. 2020, 221, 2000190.

(6) (a) Löwe, C.; Weder, C. Adv. Mater. 2002, 14, 1625. (b) Crenshaw, B. R.; Weder, C. Macromolecules 2006, 39, 9581.

(7) Donati, F.; Pucci, A.; Cappelli, C.; Mennucci, B.; Ruggeri, G. J. Phys. Chem. B 2008, 112, 3668.

(8) (a) Crenshaw, B. R.; Weder, C. Chem. Mater. 2003, 15, 4717. (b) Crenshaw, B. R.; Burnworth, M.; Khariwala, D.; Hiltner, A.; Mather, P. T.; Simha, R.; Weder, C. Macromolecules 2007, 40, 2400.

(9) Kinami, M.; Crenshaw, B. R.; Weder, C. Chem. Mater. 2006, 18, 946.

(10) Pucci, A.; Di Cuia, F.; Signori, F.; Ruggeri, G. J. Mater. Chem. 2007, 17, 783.

(11) Pucci, A.; Bertoldo, M.; Bronco, S. Macromol. Rapid Commun. 2005, 26, 1043.

(12) Lott, J.; Weder, C. Macromol. Chem. Phys. 2010, 211, 28.
(13) Lavrenova, A.; Holtz, A.; Simon, Y. C.; Weder, C. Macromol. Mater. Eng. 2016, 301, 549.

(14) (a) Ciardelli, F.; Ruggeri, G.; Pucci, A. Chem. Soc. Rev. 2013, 42, 857. (b) Schrettl, S.; Balkenende, D. W. R.; Calvino, C.; Karman, M.; Lavrenova, A.; Neumann, L. N.; Sagara, Y.; Verde-Sesto, E.; di Giannantonio, M.; Simon, Y. C.; Fromm, K. M.; Lattuada, M.; Weder, C. Chimia 2019, 73, 7.

(15) (a) Bao, S.; Li, J.; Lee, K. I.; Shao, S.; Hao, J.; Fei, B.; Xin, J. H. ACS Appl. Mater. Interfaces 2013, 5, 4625. (b) Cellini, F.; Khapli, S.; Peterson, S. D.; Porfiri, M. Appl. Phys. Lett. 2014, 105, 061907. (c) Cellini, F.; Zhou, L.; Khapli, S.; Peterson, S. D.; Porfiri, M. Mech. Mater. 2016, 93, 145.

(16) Kunzelman, J.; Gupta, M.; Crenshaw, B. R.; Schiraldi, D. A.; Weder, C. Macromol. Mater. Eng. 2009, 294, 244.

(17) Calvino, C.; Sagara, Y.; Buclin, V.; Haehnel, A. P.; Del Prado, A.; Aeby, C.; Simon, Y. C.; Schrettl, S.; Weder, C. Macromol. Rapid Commun. 2019, 40, e1800705.

(18) (a) Löwe, R.; Krüger, P.; Knebel, M.; Ehreke, J.; Pudleiner, H.; Yesildag, M. C.; Meyer, K.; Pophusen, D.; Büchner, J. US20110274883 A1, 2011. (b) Bothe, M.; Emmerling, F.; Pretsch, T. Macromol. Chem. Phys. 2013, 214, 2683. (c) Ecker, M.; Pretsch, T. RSC Adv. 2014, 4, 46680. (d) Fritzsche, N.; Pretsch, T. Macromolecules 2014, 47, 5952. (e) Mirtschin, N.; Pretsch, T. RSC Adv. 2015, 5, 46307. (f) Pudleiner, H.; Meyer, K.; Winkler, J.; Braeuer, W.; Nickel, J.; Pehlert, C. EP2850126, 2015. (g) Bouaziz, R.; Roger, F.; Prashantha, K. Smart Mater. Struct. 2017, 26, 055009. (h) Shirole, A.; Perotto, C. U.; Balog, S.; Weder, C. ACS Appl. Mater. Interfaces 2018, 10, 24829.

(19) Shirole, A.; Nicharat, A.; Perotto, C. U.; Weder, C. Macromolecules 2018, 51, 1841.

(20) (a) Martínez-Abadía, M.; Varghese, S.; Giménez, R.; Ros, M. B. J. Mater. Chem. C 2016, 4, 2886. (b) Kaneko, R.; Sagara, Y.; Katao, S.; Tamaoki, N.; Weder, C.; Nakano, H. Chemistry 2019, 25, 6162.

(21) Morbitzer, L.; Hespe, H. J. Appl. Polym. Sci. 1972, 16, 2697. 